Pontificia Universidade Católica

DO RIO DE JANEIRO

Cesar Gonzalo Vera Vasquez

Redução das Emissões em Geradores

Diesel-Gás

Dissertação de Mestrado

Dissertação apresentada como parte dos requisitos para obtenção do título de Mestre pelo Programa de Pós-Graduação em Engenharia Mecânica da PUC Rio.

Orientador: Prof. Sergio Leal Braga

Rio de Janeiro

Setembro de 2009 
Cesar Gonzalo Vera Vasquez

\section{Redução de Emissões em Geradores Diesel-gás}

Dissertação apresentada como parte dos requisitos para obtenção do título de Mestre pelo Programa de PósGraduação em Engenharia Mecânica da PUC - Rio. Aprovada pela Comissão Examinadora abaixo assinada.

Prof. Sergio Leal Braga Orientador Departamento de Engenharia Mecânica - PUC-Rio.

Prof. Ricardo Hernandez Pereira Co- Orientador Faculdade de Engenharia Mecânica - UFU-MG.

Prof. Carlos Valois Maciel Braga Departamento de Engenharia Mecânica - PUC-Rio.

Prof. José Alberto dos Reis Parise Departamento de Engenharia Mecânica - PUC-Rio.

Prof. Marcos Sebastião de Paula Gomes Departamento de Engenharia Mecânica - PUC-Rio.

Prof. José Eugênio Leal Coordenador Setorial do Centro Técnico Científico - PUC-Rio 
Todos os direitos reservados. É proibida a reprodução total ou parcial do trabalho sem autorização da universidade, do autor e dos orientadores.

\section{Cesar Gonzalo Vera Vasquez}

Formado em Engenharia Mecânica pela Universidad Nacional de San Agustín. Arequipa- Perú, em Dezembro de 2005. Todo o ano 2006 atou como engenheiro em projetos de manutenção e planejamento em diversas empresas peruanas.

Ficha Catalográfica

Vera Vasquez, Cesar Gonzalo

Redução das emissões em geradores diesel-gás / Cesar Gonzalo Vera Vasquez; orientadores: Sergio Leal Braga, Ricardo Hernandez Pereira. - Rio de Janeiro: PUC, Departamento de Engenharia Mecânica, 2009.

118 f.: il. (color.) ; $30 \mathrm{~cm}$

Dissertação (Mestrado) - Pontifícia Universidade Católica do Rio de Janeiro, Departamento de Engenharia Mecânica

Inclui bibliografia

1. Engenharia mecânica - Teses. 2. Geração termelétrica. 3. Motor diesel-gás. 4. Emissões. 5. Gás natural. 6. Combustão. I. Braga, Sergio Leal. II. Pereira, Ricardo Hernandez. III. Pontifícia Universidade Católica do Rio de Janeiro. Departamento de Engenharia Mecânica. IV. Título. 
A mis padres Reynaldo y Maruja, por el inmenso amor y apoyo siempre.

A Maricela por su inmenso cariño y estímulo en mi viaje a Brasil. 


\section{Agradecimentos}

Aos Professores Sergio Leal Braga e Ricardo Hernandez Pereira, pela confiança, paciência e apoio.

Aos meus familiares, em especial ao Arquiteto Álvaro Pilares Vera e a Licenciada Consuelo Vera Ramos pelo estímulo e ajuda na minha viagem ao Brasil.

Ao Departamento de Engenharia Mecânica da PUC-Rio, professores e funcionários.

Ao ITUC (Instituto Tecnológico da PUC-Rio), aos funcionários administrativos e funcionários das oficinas mecânicas pela ajuda subministrada.

Aos colegas Julio Cuisano Egúsquiza, Juan Milón Guzmán, Miguel Mozo Leon, Anthony Roque Ccacya, José Aguilar Franco, Fernando Ferrari, Andrea Carvalho e Filipe Alves pela parceria e amizade.

Ao engenheiro Severino Wanderley pelo suporte eletrônico e amizade.

Aos técnicos do Laboratório de Engenharia Veicular da PUC-Rio, Gilson Coutinho Pradanoff e Fabrício Ferraz Gonçalves, pelo apoio técnico na montagem experimental.

Para Gerson Silvério, pela ajuda na montagem do aparato experimental e pela amizade.

Aos amigos que fiz no Brasil: Gilmar, Jorge, Marko, David, Antonio, Miguel, Nilton, Gerardo, Johanna, Melisa, Evelyn R., Rocio Z., Mayra, Melva, Claudia, Rocio R., Marquito, César M., Carlos G., Liseth, Alejandra, Liliana, Tania e todos meus amigos da PUC-Rio pela amizade e estímulo.

Para CAPES, pelo suporte financeiro. 


\section{Resumo}

Vera Vasquez, Cesar Gonzalo; Braga, Sergio Leal; Pereira, Ricardo Hernandez. Redução das emissões em Geradores Diesel-gás. Rio de Janeiro, 2009. 118p. Dissertação de Mestrado - Departamento de Engenharia Mecânica, Pontifícia Universidade Católica do Rio de Janeiro.

O trabalho tem como objetivo a conversão de um grupo gerador, originalmente Diesel, para operar no modo Diesel-Gás, onde estes dois combustíveis são administrados simultaneamente no motor. Para tal foi utilizado um grupo gerador de 120KW, com um motor Perkins 1006 TAG (turbo alimentado com intercooler). Medidas experimentais foram realizadas tanto no modo Diesel quanto no bicombustível Diesel-gás. Foram avaliados: desempenho e, principalmente, emissões de poluentes atmosféricos. A redução das emissões foi realizada mediante a restrição parcial do ar de combustão, regulada por uma válvula tipo borboleta, que funciona eletronicamente, posicionada na entrada do coletor de admissão. A relação Diesel-Gás foi também avaliada, onde o segundo combustível era administrado através de um sistema eletrônico de injeção de gás natural. Os resultados indicam que em cargas baixas as reduções de monóxido de carbono e hidrocarbonatos são significativas (50\% de redução de HC e $20 \%$ de CO) com máximas taxas de substituição. O mesmo se observa em cargas intermediárias. Em cargas médias e baixas observa-se um leve aumento nas emissões de óxido nitroso. Pode-se observar uma melhora no rendimento global do grupo gerador com o aumento da carga e da taxa de substituição. De forma geral, conseguiu-se reduzir os níveis de emissões em altas cargas, principalmente de hidrocarbonetos e monóxido de carbono.

\section{Palavras-chave}

Geração termelétrica; Motor Diesel-Gás; Emissões; Gás Natural; Combustão. 


\section{Abstract}

Vera Vasquez, Cesar Gonzalo; Braga, Sergio Leal (Advisor); Pereira, Ricardo Hernandez (Advisor). Reduction of the Emissions in Gas Diesel Generators. Rio de Janeiro, 2009. 118p. MSc. Dissertation - Departamento de Engenharia Mecânica, Pontifícia Universidade Católica do Rio de Janeiro.

The objective of this study is converting a generator, originally Diesel, to operate in a Diesel-Gas; two fuels are administered simultaneously to the motor. One diesel generator of 120KW model Perkins 1006 TAG (powered with turbo intercooler), running on the Diesel / natural gas dual fuel mode, was tested. Experimental measurements were performed in both the Diesel and dual fuel diesel-gas. Are evaluated performances and; especially air pollutant emissions. The emission reduction was carried out by partial restriction of the combustion air with the help of an electronic throttle valve, positioned before the intake manifold. The ratio Diesel-Gas was evaluated, where the second fuel (natural gas) was administered with one electronic injection of natural gas. The results indicate that at low loads the reductions in carbon monoxide and hydrocarbons are significant (50\% reduction in HC and 20\% CO) with maximum replacement rates. Something like is observed at intermediate loads. In medium and low loads there is a slight increase in emissions of nitrous oxides. One can observe a slight increase in overall yield of the generator with the increased workload and the replacement rate; in general it was possible to reduce emissions at high loads, especially in hydrocarbons and carbon monoxide.

\section{Keywords}

Power Generation; Gas Diesel; Engine; Emissions; Natural Gas; Combustion. 


\section{Sumário}

1. Introdução

1.1. Poluentes Atmosféricos 19

1.2. Principais Poluentes Atmosféricos 20

1.3. Objetivos 22

1.4. Descrição da Tese 23

2. Revisão Bibliográfica 24

2.1. Combustão Diesel-Gás 24

2.2. Definições de Potências em Geradores 24

2.2.1. Potência Efetiva Contínua não Limitada 25

2.2.2. Potência Efetiva Contínua Limitada 26

2.3. Combustão Perfeita 27

2.4. Propriedades do Óleo Diesel 28

2.5. Propriedades do Gás Natural 29

2.6. Combustão Real 30

2.7. Formação de Poluentes em Motores Diesel-Gás 31

3. Aparato Experimental 32

3.1. Grupo Gerador 33

3.2. Governador Elétrico 34

3.3. Medição da Carga Elétrica 36

3.4. Medição do Consumo de Diesel 37

3.5. Controlador Eletrônico de Vazão Ar 38

3.6. Medição de Consumo de $\operatorname{Ar} \quad 39$

3.7. Controlador Eletrônico de Vazão de Gás 40

3.8. Medição do Consumo de Gás 41

3.9. Medição de Pressões e Temperaturas 42 
3.10. Software de Controle 43

3.11. Medição de Emissões 44

3.12. Dissipador de Carga Elétrica 45

3.13. Sistema de Compressão de Gás Natural 44

4. Metodologia e Desenvolvimento dos Ensaios 46

4.1. Modo Diesel Puro $\quad 47$

4.2. Modo Diesel - Gás 47

4.3. Diesel -Gás com Restrição de Ar 50

4.4. Redução de Dados

4.4.1. Potência elétrica Observada $\left(P_{e l}\right) \quad 51$

4.4.2. Vazão Mássica de $\operatorname{ar}\left(\dot{m}_{a r}\right) \quad 51$

4.4.3. Vazão Mássica de Gás Natural $\left(\dot{m}_{g}\right)$

4.4.4. Consumo Específico de Combustível (cec) 57

4.4.5. Rendimento Térmico $\left(\eta_{t}\right) \quad 58$

4.4.6. Eficiência Volumétrica $\left(\eta_{V}\right) \quad 59$

4.4.7. Razão Ar-Combustível (AC) 61

4.4.8. Razão de Equivalência Total $\left(R E_{T}\right) \quad 61$

4.4.9. Taxa de Substituição (TS) 62

4.4.10. Emissões Específicas 62

5. Resultados e Análises 66

5.1. Resultados no Modo Diesel Puro, Diesel-Gás e 66

Com Restrição de Ar

6. Conclusões e Recomendações $\quad 87$ 
7. Referências Bibliográficas

Anexo A: Analise da Propagação de Incerteza nos Cálculos

Anexo B: Planilhas de Medições

Anexo C: Planilhas de Incertezas 


\section{Lista de figuras}

Figura 1 - Emissões por tipo de fonte na região metropolitana

de Rio de Janeiro

Figura 2 - Esquema da instalação e montagem dos

equipamentos de ensaio

Figura 3 - Grupo gerador Perkins 1006 TAG modelo P135

Figura 4 - Governador eletrônico da Bomba de alta

Pressão de combustível

Figura 5 - Voltímetro e Amperímetro usados em conjunto

na medição da carga elétrica

Figura 6 - Medidor de vazão de Óleo Diesel 35

Figura 7 - Tambor para a medição de vazão de ar na 36

entrada do coletor de admissão

$\begin{array}{ll}\text { Figura } 8 \text { - Bico injetor } & 37\end{array}$

Figura 9 - Placa eletrônica de controle 38

Figura 10 - Tambor de medição de gás natural 38

Figura 11 - Arranjo dos transdutores de pressão e 39

tomadas de temperatura no lado direito a tomada dos sensores para a leitura de dados

Figura 12 - Software e Sistema de aquisição de dados 40

Figura 13 - Arranjo dos transdutores de pressão e tomadas 41 de temperatura; no lado direito a tomada dos sensores para a leitura de dados

Figura 14 - Software e Sistema de aquisição de dados 42

Figura 15 - Equipamento de medição de gases de escapamento 43

Figura 16 - Arranjo do dissipador de carga 44

Figura 17 - Sistema de compressão do gás natural 45

Figura 18 - Curva de potência elétrica e potência ao eixo em 67 função do consumo de óleo Diesel (Modo Diesel Puro) 
Figura 19 e 20 - Consumo específico de combustível em modo

diesel puro e em modo diesel-gás com máxima taxa de

substituição e consumo de combustível de diesel e de

gás natural, em modo diesel puro e diesel-gás com

máxima taxa de substituição para diferentes frações da Carga Prime

Figura 21 - Consumo Específico de Combustível

para diferentes porcentagens da Carga Prime em

função da Taxa de Substituição

Figura 22 - Consumo de diesel sem restrição

e com as máximas restrições de ar em função da

Fração de Carga Prime

Figura 23 - Rendimento Térmico com variações de carga

e restrição de ar em função da Taxa de Substituição

Figura 24 - Eficiência Volumétrica com variações de carga

e restrição de ar em função da Taxa de Substituição

Figura 25 - Temperatura de Escape em 60\% e 100\% de carga,

e restrição de ar em função da Taxa de Substituição

Figura 26 - Temperatura de Escape com variações de carga

76

e restrição de ar em função da Taxa de Substituição

Figura 27 - Emissões Específicas de Dióxido de

Nitrogênio com variações de carga e restrição de ar em função da Taxa de Substituição

Figura 28 - Emissões Específicas de Monóxido de Carbono

em $60 \%$ e 100\% de carga, com restrição de ar em função

da Taxa de Substituição.

Figura 29 - Emissões Específicas de Monóxido de

Carbono com variações de carga e restrição de ar em função da Taxa de Substituição

Figura 30 - Emissões Específicas de Hidrocarbonetos não queimados em $60 \%$ e 100\% de carga, com restrição de ar em função da Taxa de Substituição 
Figura 31 - Emissões Específicas de Hidrocarbonetos não queimados, com variações de carga e restrição de ar em função da Taxa de Substituição

Figura 32 - Emissões Específicas de Monóxido de Carbono queimados, com variação de Equivalência Total para diferentes restrições de ar. Operação Diesel-Gás com máxima taxa de substituição

Figura 33 - Emissões Específicas de Hidrocarbonetos não queimados, com variação de Equivalência Total para diferentes restrições de ar. Operação Diesel-Gás com máxima taxa de substituição 


\section{Lista de Tabelas}

Tabela 1 - Composição do gás

natural fornecida pela Companhia Distribuidora

de Gás do Estado do Rio de Janeiro

Tabela 2 - Comparação entre algumas características

físico-químicas do Óleo Diesel e o Gás Natural

Tabela 3 - Dados Técnicos do Grupo gerador

Tabela 4 - Dados técnicos dos transdutores

de pressão

Tabela 5 - Dados técnicos dos transdutores de pressão 


\section{Lista de Símbolos}

A - Amperagem medida na linha do trifásico.

(A/C) - Razão ar-combustível.

$\left(A / C_{g}\right)_{e}$ - Razão ar-gás estequiométrica.

$\left(A / C_{D}\right)_{e}$ - Razão diesel-gás estequiométrica

C - Carbono.

Cec - Consumo específico de combustível .

CO - Monóxido de carbono.

CO2 - Dióxido de carbono.

$\mathrm{CH}_{4}$ - Metano.

$\mathrm{C}_{2} \mathrm{H}_{6}$ - Etano.

$\mathrm{C}_{3} \mathrm{H}_{8}$ - Propano.

$\mathrm{C}_{4} \mathrm{H}_{10}$ - Butano.

$\mathrm{C}_{12} \mathrm{H}_{26}$ - Dodecano (óleo diesel leve).

$\mathrm{C}_{x} \mathrm{H}_{y}$ - Representação geral de um hidrocarboneto.

$\mathrm{C}_{d}$ - Coeficiente de descarga.

$\mathrm{d}$ - Diâmetro do orifício nos bocais (m).

EGR - Recirculação dos gases de escapamento.

F1 - Fator de conversão de concentrações em base seca para úmida.

$\mathrm{H}$ - Hidrogênio

HC - Hidrocarbonetos não queimados.

$\mathrm{H}_{2} \mathrm{O}$ - Vapor d'água.

$\mathrm{m}_{\mathrm{ar}}$ - Massa de ar.

$m_{D}$ - Massa de diesel.

$\mathrm{m}_{\mathrm{g}}$ - Massa de gás.

MP - Material particulado.

m - Vazão mássica (kg/h).

$\mathrm{N}$ - Velocidade angular (RPM).

$\mathrm{N}_{2}$ - Nitrogênio.

NO - Monóxido de nitrogênio.

$\mathrm{NO}_{2}$ - Dióxido de nitrogênio.

$\mathrm{NO}_{\mathrm{x}}$ - Óxidos de nitrogênio. 
$\mathrm{O}_{2}$ - Oxigênio.

$P$ - Potência ao eixo (kW)

$P_{\mathrm{el}}$ - Potência elétrica $(\mathrm{kW})$

$\mathrm{P}_{\mathrm{ar}}$ - Pressão barométrica $(\mathrm{kPa})$

$\mathrm{PCl}$ - Poder calorífico inferior.

$\mathrm{P}_{\text {sat }}$ - Pressão de saturação da água em temperatura ambiente $(\mathrm{kPa})$.

PPM - Partes por milhão

$R E_{T}$ - Razão estequiométrica total.

$\mathrm{R}_{\mathrm{ar}}$ - Constante do ar (kJ/kg.K).

$\mathrm{R}_{\mathrm{g}}$ - Constante do gás natural (kJ/kg.K).

$\mathrm{Re}$ - Numero de Reynolds

$\mathrm{SO}_{2}$ - Dióxido de enxofre.

TS - Taxa de substituição

$\mathrm{U}_{\mathrm{r}}$ - Umidade relativa

$V_{\text {bocal }}$ - Velocidade nos bocais ASME.

$V_{d}$ - Volume deslocado no cilindro $\left(\mathrm{m}^{3}\right)$

$\checkmark$ - Voltagem medida entre duas linhas do trifásico.

$\dot{V}$ - Vazão volumétrica

W - Umidade relativa 


\section{Subscritos e Letras Gregas}

$\mathrm{Ar}$ - Relativo à vazão mássica do ar.

D - Relativo à vazão mássica do ar.

D_O - Relativo ao consumo de Diesel original.

esc_u - Relativo à vazão do gás de escape em base úmida.

g - Relativo à vazão de gás.

$\rho$ ar - Massa específica do $\operatorname{ar}\left(\mathrm{kg} / \mathrm{m}^{3}\right)$.

$\rho_{\mathrm{g}}$ - Massa específica do gás $\left(\mathrm{kg} / \mathrm{m}^{3}\right)$.

$\Delta P$ ar - Diferencial de pressão no tambor de ar ( $\mathrm{kPa})$.

$\Delta P_{\mathrm{g}}$ - Diferencial de pressão no tambor de gás ( $\left.\mathrm{kPa}\right)$.

S - Relativo à concentração específica de emissões.

$\eta_{\text {t_D }}$ - Rendimento térmico em operação diesel puro.

$\eta_{\text {t_dual }}$ - Rendimento térmico em operação diesel-gás.

$\eta \vee-$ Eficiência volumétrica.

$\eta_{\mathrm{G}}$ - Eficiência do grupo gerador. 